\title{
O Fenômeno Didático Institucional da Rigidez e a Atomização das Organizações Matemáticas Escolares*
}

\section{The Institutional Didactic Phenomenon of Rigidity and the Atomization of School Mathematics Organizations}

\author{
Catarina Lucas* \\ Cecilio Fonseca ${ }^{* * *}$ \\ Josep Gascón ${ }^{* * * *}$ \\ José Casas $^{* * * * *}$
}

\begin{abstract}
Resumo
Nesta investigação estudamos um fenômeno didático complexo que se manifesta na desconexão dos conteúdos matemáticos que constituem o programa oficial do ensino secundário/médio e que está fortemente relacionado com a rigidez e a atomização da Matemática escolar. Para tal estudo utilizamos as ferramentas teóricas e metodológicas que nos proporciona a Teoria Antropológica do Didático (TAD) cujo objeto primário de investigação é a análise da atividade matemática escolar institucional. Assim, realizamos estudos exploratórios nos manuais escolares de Matemática do ensino secundário de Portugal e da Espanha, contrastando e comprovando cinco conjeturas relacionadas, por exemplo, com a ausência de questionamento e justificação das técnicas utilizadas. Concluimos que nestes países as praxeologias matemáticas apresentam-se desarticuladas, rígidas, e, consequentemente, surgem isoladas atividades de modelagem matemática nas instituições escolares.
\end{abstract}

Palavras-chave: Organização Matemática. Ensino médio/secundário. Teoria Antropológica do Didático (TAD).

\begin{abstract}
In this research, we studied a complex didactic phenomenon manifested on the disconnection of mathematical contents that constitute the official program of secondary education and that relates strongly to the rigidity and atomization of school mathematics. For this study, we used the theoretical and methodological tools that the Anthropological Theory of Didactic (ATD) provides us, which primary object of research is the analysis of the

\footnotetext{
Trabalho financiado pelo projeto: "La modelización matemática para la formación del profesorado de secundaria: del algebra al cálculo diferencial "(EDU2012-39312-C03-03).

*** Estudante de doutoramento do Departamento de Matemática Aplicada I, Universidade de Vigo, Espanha, financiada pela bolsa SFRH/BD/77335/2011 da FCT (Portugal),Universidade de Vigo, Espanha. Endereço para correspondência: Campus Universitario Lagoas, Marcosende, 36310, Vigo, Espanha. E-mail: catarinalucas.mail@gmail.com.

**** Doutor pela Universidade de Vigo, Espanha. Professor do Departamento de Matemática Aplicada I, Universidade de Vigo (EUIT), Espanha. Endereço para correspondência: Industrial, Torrecedeira 86, 36208 Vigo. Espanha. E-mail: cfonseca@uvigo.es.

Doutor pela Universidade Autónoma de Barcelona (UAB), Espanha. Professor do Departamento de Matemáticas da UAB, Facultad de Ciencias, Barcelona, Espanha. Endereço para correspondência: Edificio C, Campus de la UAB, 08193, Bellaterra, Cerdanyola del Vallès, Barcelona, España. E-mail: gascon@ mat.uab.cat.

Doutor pela Universidade de Vigo, Espanha. Professor do Departamento de Matemática Aplicada I, Universidade de Vigo, Espanha. Endereço para correspondência: E. E. Forestal, Campus Universitario A xunqueira, 36005, Pontevedra, Espanha. E-mail: jmcasas@uvigo.es.
} 
institutional school mathematics activity. Thus, we carry out exploratory studies in mathematics textbooks in Portugal and Spain secondary education, contrasting and proving five conjectures related, for example, with the absence of questioning and justification of the used techniques. We conclude that in these countries, the mathematical praxeologies are often disjointed, rigid and, consequently, there are isolated activities of mathematical modelling in educational institutions.

Keywords: Mathematical organization. Secondary education. Anthropological Theory of Didactic (ATD).

\section{Introdução: rigidez versus flexibilidade}

Neste trabalho estudaremos a rigidez da Matemática escolar utilizando as ferramentas teóricas e metodológicas que a Teoria Antropológica do Didático (TAD) nos proporciona. Em coerência com esta teoria didática, e tal como explicaremos, em seguida, detalhadamente, tomaremos um ponto de vista epistemológico e institucional em vez de cognitivo e pessoal (GASCÓN 1998, 2003).

O fenômeno da rigidez e as suas diferentes manifestações têm sido estudados por diferentes teorias didáticas segundo uma abordagem cognitiva, utilizando a noção de atividade matemática flexível, autónoma e aberta (em oposição a rígida, dirigida e rotineira). No âmbito destas abordagens, a origem do problema reside na constatação das dificuldades, contradições, confusões, obstáculos cognitivos e, em geral, fenômenos (cognitivos) que aparecem na transição do Elementary Mathematical Thinking (EMT) ao Advanced Mathematical Thinking (AMT). No início da década de 90, do século passado, alguns estudos revelaram que essa transição não poderia ser explicada exclusivamente por dificuldades na aprendizagem formal de conceitos matemáticos, mas que se deveria enfatizar especialmente o novo tipo de raciocínio matemático associado. Tommy Dreyfus constatou que os alunos do ensino primário e secundário aprendiam, na disciplina de Matemática, um grande número de procedimentos padronizados e uma grande quantidade de conhecimentos, mas praticamente nada referente à metodologia de trabalho dos matemáticos. Em particular, os alunos não aprendiam a usar os seus conhecimentos matemáticos de forma flexível para resolver problemas de um tipo desconhecido para eles (DREYFUS, 1991).

A noção de pensamento matemático flexível pode ser descrita a partir de noções mais primitivas que Tall (1996) tomou originalmente de Piaget (1972) e de trabalhos que interpretam a obra deste, como os de Dubinsky (1991) e Sfard (1991). Estas noções são as de processos mentais (ou sistemas de ações interiorizados) e de conceitos produzidos pelo encapsulamento de processos. Os conceitos assim obtidos são objetos sobre os quais se pode aplicar, por sua vez, um sistema de ações que pode ser novamente interiorizado e dar lugar a um processo mental de nível superior suscetível de ser, de novo, encapsulado num conceito 
de ordem superior e, assim sucessivamente. Gray e Tall (1994) designaram por procept uma combinação de processo e conceito produzido pelo processo, que foram representados conjuntamente por um mesmo símbolo matemático, destacando assim a natureza dual dos objetos matemáticos e o papel do simbolismo matemático no encapsulamento (de processos em objetos) (TALL, 1996).

As três noções básicas do Cálculo: função, derivada e integral (bem como a noção fundamental de limite) são exemplos de procepts. O estudo do Cálculo Elementar exige portanto, desde o início, flexibilidade suficiente para lidar com um mesmo símbolo, quer seja como representante de um processo que atua sobre determinados objetos, quer seja uma entidade singular à qual se pode aplicar outros processos para obter novos objetos. O poder do AMT surge justamente no uso flexível da estrutura dual dos objetos matemáticos citados (e dos construídos a partir deles) possibilitada, em parte, pela ambiguidade da notação usada. A rigidez dos procedimentos padronizados que caracterizam o EMT é, por conseguinte, um obstáculo cognitivo muito importante e explica muitos dos erros conceituais extravagantes (DREYFUS, 1991), apresentados pela grande maioria dos estudantes no seu primeiro encontro com o Cálculo. Relativamente a este problema, Silva et al. (1999) referiram que a aprendizagem da Matemática deveria incluir oportunidades para os alunos se envolverem em momentos genuínos de atividade matemática. Salientaram que as investigações matemáticas deveriam merecer um lugar de destaque, uma vez que: por um lado permitem a formulação de conjeturas, a avaliação da sua plausibilidade e a escolha dos testes adequados para a sua validação ou rejeição; e, por outro lado, permitem procurar argumentos que demonstrem as conjeturas que resistiram a sucessivos testes e levantar novas questões para investigar. Assim, propuseram a criação de um contexto de aula propício ao diálogo, em que o professor lança boas questões para trabalho prático com informação mínima e em que, após alguma discussão, os alunos partem para formas de trabalho de tipo exploratório, formulação de problemas, investigações ou pequenos projetos que o professor acompanha e incentiva, assumindo, num momento posterior, a coordenação da sistematização do trabalho desenvolvido e/ou da formalização de aspetos matemáticos inerentes. Os referidos autores realçaram também que a visão tradicional de uma matemática rígida, na qual as definições têm um carácter absoluto, aparece oposta àquela que as tarefas de investigação, se aceites com as suas características próprias, podem veicular.

De acordo com João Pedro da Ponte e João Filipe Matos, consideramos (e mostraremos que esta afirmação se pode sustentar empiricamente) que muitas das dificuldades que apresentam os alunos para trabalhar com tarefas de investigação e, em 
particular, para levantar questões pertinentes no desenvolvimento de tais tarefas, provêm do caráter formal da Matemática escolar e da forma como esta está organizada, pois “ [...] ensinam-se 'respostas' sem dar a mínima importância às 'questões' que as originam ou à forma como foram alcançadas" (PONTE; MATOS, 1996, p. 123).

Para estes autores, a forma como os alunos concebem as representações e notações matemáticas adequadas às situações ou fenômenos que lhes são apresentados é um elemento fundamental para a realização de investigações. Muitas vezes, os alunos manifestam ter dificuldade em conceber alguma representação, não concebem as mais adequadas, ou saltitam entre diferentes representações, o que lhes cria sérias dificuldades na realização das tarefas propostas.

No mesmo sentido, Michèle Artigue (1998) considerou que a flexibilidade na utilização de diversos registros de representações (gráficos, simbólicos, linguagem natural, gestual,...), bem como, a flexibilidade na articulação sistemática de diferentes interpretações do mesmo objeto matemático são condições essenciais para desenvolver uma atividade matemática genuína. As instituições educativas deveriam ter, sob a sua responsabilidade, o trabalho de possibilitar e capacitar a articulação de vários registros de representação e as diferentes interpretações dos objetos matemáticos, uma vez que, quando esta articulação é deixada para o trabalho privado do aluno, as possibilidades de insucesso são elevadas. Em particular, Artigue afirmou, segundo Tall (1996), que a tecnologia da informação, se usada adequadamente, pode desempenhar um papel decisivo no desenvolvimento de articulações flexíveis e no equilíbrio entre registros algébricos e gráficos.

Em seguida, e utilizando as ferramentas que nos proporciona a TAD, analisaremos detalhadamente alguns aspetos da forma de organizar as matemáticas nas instituições escolares. Isto com o objetivo de fornecer uma base empírica para sustentar e permitir especificar a hipótese (compartilhada, como já vimos, por múltiplos investigadores), segundo a qual, a Matemática escolar no ensino secundário/médio está organizada de forma rígida e atomizada, o que dificulta enormemente o desenvolvimento de uma verdadeira atividade matemática pelos estudantes. Além disso, esta investigação pretende, em primeiro lugar, suportar empiricamente que esta rigidez e atomização das matemáticas escolares constituem um fenômeno didático de caráter institucional (e não pessoal) relativamente independente das características pessoais dos sujeitos do processo didático (alunos e professores) e, até mesmo, das culturas pedagógicas nas quais eles estão imersos.

\section{Modelo epistemológico-didático proposto pela teoria antropológica do didático}


O Programa Epistemológico de Investigação em Didática da Matemática (GASCÓN 1998, 2003) surgiu da convicção de que a origem do problema da Educação Matemática está na própria Matemática. O nascimento deste Programa de Investigação ${ }^{1}$ constitui uma resposta à visível insuficiência dos modelos epistemológicos da Matemática, incluindo os modelos desenvolvidos pela epistemologia clássica, para enfrentar o Problema da Educação Matemática. O questionamento da transparência do matemático e claro pressuposto de que $o$ mistério permanece na própria Matemática, permite tomar a atividade matemática como o objeto primário do estudo, como uma nova porta de entrada da análise didática.

Para analisar a atividade matemática institucionalizada, a Teoria Antropológica do Didático (adiante, TAD), situada dentro do Programa Epistemológico, propõe inicialmente um modelo epistemológico geral da Matemática que descreve o saber matemático em termos de organizações ou praxeologias matemáticas institucionais (CHEVALLARD, 1999). Neste modelo o conhecimento matemático surge organizado em dois níveis. O primeiro nível designa-se por práxis (ou saber-fazer), refere-se à prática realizada e, por sua vez, descreve-se em dois componentes: os tipos de problemas/tarefas que se estudam e as técnicas que são usadas para os/as resolver. O segundo nível contém a parte descritiva, organizadora e justificadora da atividade, designa-se por logos (ou simplesmente, por saber) e contém o discurso matemático-racional sobre a referida prática. Analogamente, este segundo nível estrutura-se em duas componentes: a tecnologia (discurso matemático diretamente relacionado com a prática) que serve para tornar as técnicas inteligíveis, para as descrever, interpretar, justificar o seu funcionamento e, em última análise, fundamentar a produção de novas técnicas; e a teoria que dá significado aos problemas propostos, permite explicar e interpretar as descrições e justificações tecnológicas. Assim, a teoria poderá ser interpretada, em certo sentido, como uma tecnologia da tecnologia.

Ao juntar os dois níveis de atividade (práxis+logos) surge a noção de praxeologia matemática (adiante, PM) que constitui a noção básica do modelo epistemológico que propõe a TAD. Os tipos de tarefas, técnicas, tecnologias e teorias são as quatro categorias de elementos que compõem uma organização ou praxeologia matemática.

A TAD postula que toda a atividade matemática pode ser interpretada como uma atividade de produção de praxeologias com o objetivo de responder a determinadas questões

\footnotetext{
${ }^{1}$ Geralmente, considera-se que os trabalhos iniciais de Guy Brousseau e, em especial, os que abordam a epistemologia experimental, constituem o germe do Programa Epistemológico. Em Brousseau (1997) encontrase uma coleção de trabalhos seus publicados entre 1970 e 1990.
} 
problemáticas. Esta atividade requer que o estudante (seja este um aluno, um professor ou um investigador) disponha de técnicas matemáticas adequadas e que possa usar, quando requerido, um discurso matemático para interpretar, dar sentido e desenvolver a prática matemática. A atividade de resolver problemas fica assim integrada indissoluvelmente nas restantes componentes da PM e, assim, não poderá interpretar-se separadamente das técnicas matemáticas que estão disponíveis numa determinada instituição, nem de forma independente do discurso matemático disponível.

Esta integração indissolúvel das componentes de uma PM reflete-se no tipo de atividade didática, ou seja, na atividade de estudo e de auxílio ao estudo da Matemática que propõe a TAD (CHEVALLARD; BOSCH; GASCÓN, 1997). Assim, com efeito, no modelo didático proposto por esta teoria, a atividade de desenvolver técnicas matemáticas adequadas para abordar as tarefas que aparecem ao longo do processo de estudo, bem como, a de construir o discurso matemático que as justifica e que permite construir novas técnicas, não se situa sob a exclusiva responsabilidade do professor (ou do diretor do processo de estudo).

Em resumo, o modelo epistemológico-didático que propõe a TAD, embora salientando o papel central da atividade de resolução de tipos de problemas por parte dos estudantes, enfatiza que tal atividade não consiste em solucionar problemas a partir de técnicas matemáticas dadas e no âmbito de uma teoria matemática predeterminada. Por outro lado, a construção destas técnicas e o seu desenvolvimento progressivo, bem como, a construção de um discurso teórico justificativo e interpretativo da prática matemática, formam uma parte essencial do trabalho de resolução de problemas. Até mesmo, a abordagem de novos problemas matemáticos depende muitas vezes do questionamento das técnicas e de certos aspetos do discurso teórico.

$\mathrm{Na}$ verdade, o matemático não ansia apenas criar bons problemas e resolvê-los, mas pretende, além disso, caracterizar, delimitar e inclusivamente classificar os problemas em "tipos de problemas". Para avançar no seu trabalho, o matemático necessita de construir, desenvolver e caracterizar as técnicas que utiliza para resolver os problemas, até ao ponto de controlá-las e padronizar o seu uso, pelo que deve estabelecer as condições sob as quais as técnicas funcionam ou deixam de ser aplicáveis e, em última análise, construir argumentos sólidos e eficazes que sustentem a validade dos seus procedimentos.

Surge assim, uma dimensão da atividade matemática desconhecida na Matemática escolar (incluindo a Matemática universitária) que denominamos por questionamento tecnológico das técnicas matemáticas que se utilizam. Esta dimensão contém todas as questões problemáticas que aparecem quando se consideram as técnicas como objetos de 
estudo em si mesmas, em vez de tratá-las como se fossem dadas antecipadamente, como se fossem transparentes ou inquestionáveis e como se a única atividade que poderia ser realizada com elas fosse a resolução de problemas. Na verdade, na Matemática escolar habitual, tudo está preparado para que as técnicas funcionem bem sempre que se necessite delas, e para que não haja nenhum conflito entre as técnicas disponíveis e as tarefas matemáticas propostas.

Com a integração do questionamento tecnológico na atividade matemática, as próprias técnicas são tomadas como objeto de estudo, os problemas matemáticos podem utilizar-se como um meio para colocar em causa a economia, a eficácia, a fiabilidade e o âmbito de aplicabilidade das técnicas matemáticas.

\section{Aspetos da rigidez e a atomização das organizações matemáticas do secundário}

A tese de Fonseca (2004) revela a atomização das organizações matemáticas e a rigidez no tipo de tarefas e técnicas que os estudantes utilizam no ensino secundário espanhol, mostrando a ausência escolar do questionamento tecnológico das técnicas matemáticas, ou seja, a ausência institucional de uma análise do custo, da fiabilidade e do domínio de validade das diferentes técnicas úteis para executar uma tarefa, que poderia permitir flexibilizar a atividade matemática escolar (FONSECA, 2004; BOSCH; FONSECA; GASCÓN, 2004).

Assim, no ensino secundário, a Matemática surge como uma sequência de conhecimentos pontuais, que consiste basicamente em aplicar técnicas predeterminadas para um certo tipo de problemas, após uma apresentação teórica descritiva por parte do docente, em que raramente é questionada a necessidade de justificar a técnica usada para a atividade matemática, nem o seu domínio de validade. Para resolver este problema, a TAD sugere a introdução de um trabalho prolongado de modelagem de situações matemáticas ou extramatemáticas capazes de gerar o desenvolvimento de novas técnicas pelos alunos na atividade matemática escolar. Usando a noção de praxeologia matemática pontual e local ${ }^{2}$, elaboramos uma conjetura geral como base para reformular o problema docente como um verdadeiro problema de investigação didática no âmbito do Programa Epistemológico de Investigação em Didática da Matemática:

No Secundário o estudo das PM foca o bloco técnico-prático com baixa incidência do bloco tecnológico-teórico sobre a atividade matemática. Há uma ausência de qualquer questionamento tecnológico dos tipos de tarefas e de

\footnotetext{
2 As PM mais elementares chamam-se pontuais e são constituídas em torno do que, numa determinada instituição, é considerado um único tipo de tarefas. Quando uma PM é obtida por integração de um determinado conjunto de PM pontuais, tais que todas aceitam um discurso tecnológico comum, diremos que temos uma PM local caracterizada por tal tecnologia. Na TAD também se fala de PM "regionais" e "globais" (CHEVALLARD, 1999).
} 
técnicas matemáticas. Assim, as PM no Secundário são pontuais, muito rígidas e isoladas (ou pouco coordenadas entre si), o que dificulta, e inclusivamente impede, que nesta instituição se reconstruam efetivamente PM locais que integrem de maneira flexível as PM pontuais.

Quadro 1 - Problema de investigação didática (FONSECA, 2004)

A partir desta conjetura geral, propomos cinco conjeturas específicas relativas à atividade matemática escolar do ensino secundário (FONSECA, 2004, p.45-48):

C1. As técnicas matemáticas dependem fortemente da nomenclatura

C2. A aplicação de uma técnica não implica a interpretação do resultado obtido

C3. Cada tarefa está associada a uma técnica privilegiada

C4. Não há reversão das técnicas para realizar a tarefa matemática inversa

C5. Ausência de situações abertas de modelagem

É importante sublinhar que esta quinta conjetura terá um papel especial visto que, em certo sentido, contém as restantes, uma vez que estas podem ser interpretadas como restrições institucionais que tornam difícil e, inclusivamente, impedem a vida da modelagem matemática nas instituições escolares.

O primeiro objetivo deste trabalho, mais do que comparar a situação de desarticulação e fragmentação da Matemática em Portugal com a da Espanha ${ }^{3}$, é o de estudar em que medida e em que sentido o fenômeno didático-matemático de desarticulação e correspondente rigidez das PM escolares é generalizável para além das instituições escolares espanholas e, portanto, apresenta um caráter institucional em vez de pessoal. Pretendemos, mesmo assim, analisar algumas das principais consequências deste fenômeno didático nas atuais instituições escolares em relação à possibilidade de existência da atividade de modelagem matemática em tais instituições. Para o contraste experimental dessas conjeturas elegemos três tipos de dados empíricos como indicadores das características das PM que se reconstroem nas duas instituições:

1) Os programas oficiais e respectivos desenhos curriculares de Matemática, em particular, do $3^{\circ}$ ciclo e ensino secundário português e do ensino secundário obrigatório e bacharelato do sistema escolar espanhol.

2) As respostas de uma amostra de estudantes de Matemática de escolas portuguesas e espanholas a tarefas matemáticas propostas num questionário (LUCAS, 2010).

3) Os dados obtidos a partir da análise dos tipos de tarefas que propõe uma amostra de manuais escolares aprovados oficialmente pelas autoridades educacionais portuguesa

\footnotetext{
${ }^{3}$ Em investigações futuras gostaríamos de recolher e analisar dados de outros países com o intuito de verificar se o fenômeno da rigidez e incompletude das matemáticas é independente das tradições culturais, da sociedade em estudo, ou mesmo, do nível de escolaridade em análise.
} 
e espanhola para uso nos níveis educativos acima referidos. Estes dados podem ser considerados como a resposta dos livros didáticos ao citado questionário.

Por uma questão de espaço, neste trabalho explicitaremos apenas os dados empíricos referentes aos manuais escolares (ponto 3) e descreveremos parcialmente, nas conclusões, alguns dos resultados obtidos tomando como base empírica as respostas facultadas por uma ampla amostra de estudantes a um questionário (ponto 2), com o objetivo de sublinhar a coerência entre os dados obtidos em ambos os casos. Os detalhes complementares deste estudo podem ser consultados em Lucas (2010). Num segundo momento, pretendemos explicar as diferenças mais significativas nos currículos e livros didáticos dos sistemas escolares de Portugal e da Espanha.

\section{Indicador empírico: os manuais escolares}

O manual escolar ou livro didático é uma publicação especializada, com identidade própria, que nasceu como resposta às necessidades do sistema educativo geral público e do modelo de ensino simultâneo. Logo, podemos afirmar que este indicador empírico representa muito bem o saber institucional tal como surge no sistema escolar e que, através da sua metamorfose, constitui um material constante do sistema didático que condiciona fortemente o tipo de atividade matemática que se desenvolve na sala de aula.

Neste estudo analisamos livros didáticos que exploram o currículo oficial do $3^{\circ}$ ciclo e do ensino secundário português e, posteriormente, comparamos os resultados obtidos com os dados relativos ao ensino secundário obrigatório e bacharelado espanhol apresentados em Fonseca (2004). A seleção dos manuais tomou em consideração a sua ampla difusão nas instituições escolares do país. Assim, foram analisados dois manuais de cada ano de escolaridade descritos em Lucas (2010).

Seguidamente apresenta-se uma síntese dos resultados agrupados por conjeturas:

\section{C1. As técnicas matemáticas dependem fortemente da nomenclatura}

Propomos uma especificação desta conjetura para quatro temas específicos: Derivação, Limites, Representação gráfica de funções elementares e Álgebra (em particular, equações do segundo grau completas), conforme mostra a tabela seguinte:

Tabela 1 - Especificação da conjetura 1 e respectivos resultados 


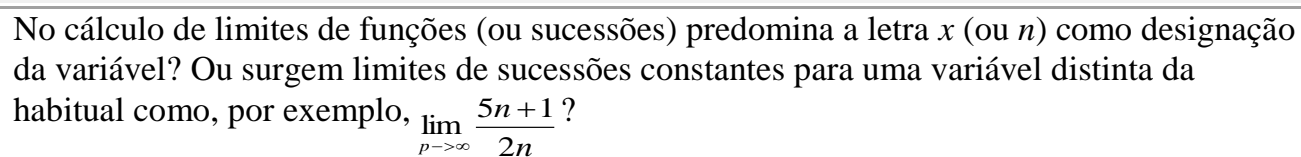

No cálculo de derivadas predomina a letra $x$ como denominação da variável independente real?

Na representação gráfica de funções predomina a letra $x$ como denominação da variável independente real?

$\mathrm{Na}$ resolução de equações do segundo grau, pela fórmula, predomina a letra $x$ como denominação da variável independente real?
C1A

C1B

C1C

C1D
Portugal

\begin{tabular}{|c|c|c|c}
\cline { 3 - 3 } \multicolumn{2}{c}{} & \multicolumn{2}{c}{$\begin{array}{c}\text { Número de } \\
\text { exercícios }\end{array}$} \\
\hline & Tipo de tarefas & $\begin{array}{c}\text { Variável } \\
\boldsymbol{x} / \boldsymbol{n}\end{array}$ & $\begin{array}{c}\text { Variável } \\
\text { distinta } \\
\text { de } \boldsymbol{x} / \boldsymbol{n}\end{array}$ \\
\hline C1A & Cálculo de limites & 329 & 0 \\
\hline C1B & Cálculo de derivadas & 243 & 14 \\
\hline C1C & Gráficos de funções & 211 & 38 \\
\hline C1D & Fórmula resolvente & 82 & 15 \\
\hline
\end{tabular}

Fonte: Lucas (2010)
Espanha $^{4}$

\begin{tabular}{|c|c|c|c|}
\hline & \multicolumn{2}{|c|}{ Número de exercícios } \\
\hline & $\begin{array}{l}\text { Tipo de } \\
\text { tarefas }\end{array}$ & $\begin{array}{c}\text { Variável } \\
x / n\end{array}$ & $\begin{array}{c}\text { Variável } \\
\text { distinta } \\
\text { de } x / n\end{array}$ \\
\hline C1B & $\begin{array}{l}\text { Cálculo de } \\
\text { derivadas }\end{array}$ & 952 & 5 \\
\hline C1C & $\begin{array}{l}\text { Gráficos de } \\
\text { funções }\end{array}$ & 492 & 2 \\
\hline
\end{tabular}

Fonte: Fonseca (2004)

As tabelas referem-se ao número total de tarefas de cada tipo que aparecem no conjunto dos manuais analisados. Assim, por exemplo, no conjunto de manuais portugueses analisados surgem 243 tarefas referentes ao cálculo de derivadas relativamente à variável $x \mathrm{e}$ apenas 14 tarefas desse tipo propõem a utilização de uma variável diferente de $x$. Analogamente, no conjunto de manuais espanhóis analisados, contabilizamos 952 tarefas referentes ao cálculo de derivadas relativamente à variável $x$ e somente 5 tarefas desse tipo usam uma variável distinta de $x$.

\section{C2. A aplicação de uma técnica não implica a interpretação do resultado obtido}

Nos livros didáticos, para cada tipo de tarefa, contamos, por um lado, o número de exercícios que envolvem a interpretação da técnica ou do resultado e, por outro lado, o número de exercícios que não incluem.

Tabela 2 - Especificação da conjetura 2 e respectivos resultados

\footnotetext{
${ }^{4}$ Dos resultados do estudo efetuado na Espanha, não apresentamos aqui os referentes às conjeturas C1A e C1D porque estão relacionados com o cálculo de integrais e com a racionalização de denominadores que são temas não abordados no ensino secundário português. Salientamos que o estudo efetuado em Portugal é uma ampliação do estudo espanhol e que o principal objetivo deste trabalho não é comparar os resultados obtidos nos dois países mas sim, à semelhança de Fonseca (2004), constatar o fenômeno de rigidez e desarticulação das matemáticas no sistema de ensino português.
} 


\begin{tabular}{|l|r|}
\hline Bloco 2 & Conjetura \\
\hline \begin{tabular}{l|c|}
\hline O cálculo do limite de uma função, dada pela sua expressão analítica, inclui a \\
interpretação do resultado?
\end{tabular} & C2A \\
\hline $\begin{array}{l}\text { O cálculo da derivada de uma função inclui a interpretação do resultado como } \\
\text { variação da função? }\end{array}$ & C2B \\
\hline O estudo da continuidade de uma função inclui a interpretação do resultado? & C2C \\
\hline O cálculo da derivada de uma função inclui a interpretação física do resultado? & C2D \\
\hline \begin{tabular}{l|l|} 
Determinar o limite de uma função num problema de modelagem inclui a \\
interpretação do resultado no contexto real?
\end{tabular} & C2E \\
\hline
\end{tabular}

Portugal

\begin{tabular}{|c|c|c|c|}
\hline & Tipo de tarefas & \multicolumn{2}{|c|}{ Número de Exercícios } \\
\hline $\mathrm{C2A}$ & $\begin{array}{l}\text { Cálculo do limite } \\
\text { de uma função }\end{array}$ & $\begin{array}{c}\text { sem interp. } \\
329\end{array}$ & $\begin{array}{c}\text { com interp. } \\
0\end{array}$ \\
\hline C2B & $\begin{array}{c}\text { Cálculo da } \\
\text { derivada de uma } \\
\text { função }\end{array}$ & $\begin{array}{c}\text { sem interp. } \\
\text { como variação } \\
463\end{array}$ & $\begin{array}{c}\text { com } \\
\text { interp.como } \\
\text { variação } \\
40\end{array}$ \\
\hline $\mathrm{C2C}$ & $\begin{array}{c}\text { Estudo da } \\
\text { continuidade de } \\
\text { uma função }\end{array}$ & $\begin{array}{c}\text { sem interp. } \\
435\end{array}$ & $\begin{array}{c}\text { com interp. } \\
79\end{array}$ \\
\hline C2D & $\begin{array}{c}\text { Cálculo da } \\
\text { derivada de uma } \\
\text { função } 5 \\
\end{array}$ & $\begin{array}{l}\text { sem interp. } \\
\text { física } \\
300 \\
\end{array}$ & $\begin{array}{c}\text { com interp. } \\
\text { física } \\
4 \\
\end{array}$ \\
\hline C2E & $\begin{array}{l}\text { Cálculo do limite } \\
\text { de uma função em } \\
\text { contexto real }\end{array}$ & $\begin{array}{l}\text { sem interp. } \\
\text { em contexto } \\
\text { real } \\
85\end{array}$ & $\begin{array}{c}\text { com interp.em } \\
\text { contexto real } \\
0\end{array}$ \\
\hline
\end{tabular}

Fonte: Lucas (2010)
Espanha $^{6}$

As tabelas refletem claramente a diferença nos manuais consultados entre o número de exercícios propostos para serem resolvidos de forma mecânica e a ausência quase absoluta de exercícios que exigem a interpretação do resultado.

\section{C3. A cada tarefa está associada uma técnica privilegiada}

Ao analisar os manuais contamos quantos exercícios de realização de uma tarefa específica incluem uma única técnica e quantos sugerem a resolução da tarefa por uma técnica diferente.

\footnotetext{
${ }^{5}$ A análise deste tipo de tarefa nos manuais de Portugal envolveu apenas os exercícios que surgem depois do estudo da interpretação física da derivada de uma função (como velocidade ou aceleração) nos ditos manuais. Daí só terem sido analisadas 304 tarefas de um total de 503 tarefas referentes ao cálculo da derivada de uma função.

${ }^{6}$ Dos resultados do estudo efetuado na Espanha, não apresentamos aqui os referentes às conjeturas C2C, C2D e $\mathrm{C} 2 \mathrm{E}$ porque estão relacionados com temas não abordados no ensino secundário português. Ver nota de rodapé da Tabela 1.
} 
Tabela 3 - Especificação da conjetura 3 e respectivos resultados

\begin{tabular}{|c|c|}
\hline Bloco 3 & Conjetura \\
\hline $\begin{array}{l}\text { A técnica algébrica para determinar a derivada de uma função num ponto é } \\
\text { mais frequente que a técnica geométrica (calcular o declive da reta tangente)? }\end{array}$ & $\mathrm{CBA}$ \\
\hline $\begin{array}{l}\text { No cálculo do valor final obtido diminuindo ou aumentando uma certa } \\
\text { percentagem do valor inicial, é requerida mais do que uma técnica? }\end{array}$ & C3B \\
\hline $\begin{array}{l}\text { No cálculo da derivada de uma função dada analiticamente predomina uma } \\
\text { técnica específica para cada tipo de função, por exemplo, a regra do quociente } \\
\text { para funções racionais? }\end{array}$ & C3C \\
\hline $\begin{array}{l}\text { Na resolução de inequações predomina a técnica algébrica (estudo algébrico } \\
\text { do sinal da função) sobre a técnica baseada no estudo gráfico da função } \\
\text { associada? }\end{array}$ & C3D \\
\hline
\end{tabular}

Portugal

Espanha $^{7}$

\begin{tabular}{|c|c|c|c|}
\hline & \multirow{2}{*}{$\begin{array}{l}\text { Tipo de } \\
\text { tarefas }\end{array}$} & \multicolumn{2}{|c|}{ Exercícios de realização } \\
\hline & & $\begin{array}{c}\text { com uma só } \\
\text { técnica }\end{array}$ & $\begin{array}{l}\text { com mais de } \\
\text { uma técnica }\end{array}$ \\
\hline C3B & $\begin{array}{c}\text { Cálculo } \\
\text { percentagens }\end{array}$ & 112 & 2 \\
\hline $\mathrm{C3C}$ & $\begin{array}{c}\text { Cálculo } \\
\text { algébrico de } \\
\text { derivadas }\end{array}$ & 262 & 9 \\
\hline \multirow[t]{2}{*}{ C3D } & \multirow{2}{*}{$\begin{array}{c}\text { Resolução } \\
\text { de } \\
\text { inequações } \\
\text { de grau } \geq 2\end{array}$} & Algebricamente & Graficamente \\
\hline & & 28 & 33 \\
\hline C3A & $\begin{array}{l}\text { Cálculo da } \\
\text { derivada } \\
\text { num ponto }\end{array}$ & 435 & 80 \\
\hline
\end{tabular}

\begin{tabular}{|c|c|c|c|}
\hline & \multirow{2}{*}{$\begin{array}{l}\text { Tipo de } \\
\text { tarefas }\end{array}$} & \multicolumn{2}{|c|}{ Exercícios de realização } \\
\hline & & $\begin{array}{l}\text { com uma só } \\
\text { técnica }\end{array}$ & $\begin{array}{l}\text { com mais de } \\
\text { uma técnica }\end{array}$ \\
\hline C3B & \begin{tabular}{|c|} 
Cálculo \\
percentagens
\end{tabular} & 43 & 37 \\
\hline $\mathrm{C} 3 \mathrm{C}$ & $\begin{array}{c}\text { Cálculo de } \\
\text { derivadas }\end{array}$ & 952 & 8 \\
\hline \multirow[t]{2}{*}{ C3D } & \multirow{2}{*}{\begin{tabular}{|c|}
$\begin{array}{c}\text { Resolução } \\
\text { de } \\
\text { inequações } \\
\text { quadráticas }\end{array}$ \\
\end{tabular}} & \multicolumn{2}{|c|}{ Algebricamente Graficamente } \\
\hline & & 25 & 4 \\
\hline
\end{tabular}

Fonte: Fonseca (2004)

Fonte: Lucas (2010)

Os dados mostram claramente o grande número de exercícios propostos para resolver uma tarefa por uma única técnica (a privilegiada) e a menor quantidade de exercícios que visam resolver uma tarefa por mais de uma técnica ou por uma técnica diferente da considerada privilegiada.

\section{C4. Não há reversão das técnicas para realizar a tarefa matemática inversa}

$\mathrm{Na}$ análise dos manuais contamos, para cada tema específico, os exercícios relacionados com a tarefa direta e com a tarefa inversa. Nesta quarta conjetura as hipóteses coincidem nos estudos efetuados nos dois países, portanto comparamos todos os resultados obtidos apresentados nas tabelas seguintes:

\footnotetext{
${ }^{7}$ Dos resultados do estudo efetuado na Espanha, não apresentamos aqui os referentes à conjetura C3A por esta ser relativa a um tema não abordado no ensino secundário português. Ver nota de rodapé da Tabela 1.
} 
Tabela 4 - Especificação da conjetura 4 e respectivos resultados

\begin{tabular}{|c|c|}
\hline \multicolumn{1}{|c|}{ Bloco 4} & Conjetura \\
\hline $\begin{array}{c}\text { Na representação de funções }{ }^{8} \text { predomina a tarefa de representar graficamente a função a } \\
\text { partir da expressão analítica sobre a tarefa inversa: obter uma expressão analítica a partir } \\
\text { do gráfico da função? }\end{array}$ & C4A \\
\hline $\begin{array}{c}\text { No estudo de funções polinomiais, nos manuais surge a tarefa de determinar os zeros de } \\
\text { uma função a partir da sua expressão. Será que aparece a tarefa inversa: descobrir uma } \\
\text { função polinomial dadas as suas raízes? }\end{array}$ & C4B \\
\hline $\begin{array}{c}\text { Nos sistemas de equações, predomina a tarefa de resolver sistemas (tarefa direta) sobre a } \\
\text { tarefa inversa: descobrir sistemas de equações (algebricamente ou geometricamente) dadas } \\
\text { as soluções? }\end{array}$ & C4C \\
\hline $\begin{array}{c}\text { No trabalho com diferentes linguagens, predomina a tradução de linguagem natural para } \\
\text { algébrica (tarefa direta) sobre a tradução inversa de uma expressão algébrica para a } \\
\text { linguagem verbal }\end{array}$ & C4D \\
\hline
\end{tabular}

Portugal

\begin{tabular}{|c|c|c|}
\hline & $\begin{array}{l}\text { TAREFA } \\
\text { DIRETA }\end{array}$ & $\begin{array}{l}\text { TAREFA } \\
\text { INVERSA }\end{array}$ \\
\hline & $\begin{array}{l}\text { Representar } \\
\text { graficamente a } \\
\text { partir da } \\
\text { expressão } \\
\text { analítica }\end{array}$ & $\begin{array}{c}\text { Escrever } \\
\text { analiticamente } \\
\text { uma função a } \\
\text { partir do gráfico }\end{array}$ \\
\hline \multirow[t]{2}{*}{$\mathrm{C} 4 \mathrm{~A}$} & 212 & 59 \\
\hline & $\begin{array}{l}\text { Resolver uma } \\
\text { equação } \\
\text { polinomial }\end{array}$ & $\begin{array}{c}\text { Determinar uma } \\
\text { equação } \\
\text { polinomial dadas } \\
\text { as raízes } \\
\end{array}$ \\
\hline \multirow[t]{2}{*}{$\mathrm{C} 4 \mathrm{~B}$} & 78 & 28 \\
\hline & $\begin{array}{c}\text { Resolver um } \\
\text { sistema de } \\
\text { equações lineares }\end{array}$ & $\begin{array}{c}\text { Determinar um } \\
\text { sistema de } \\
\text { equações lineares } \\
\text { dadas as soluções }\end{array}$ \\
\hline \multirow[t]{2}{*}{$\mathrm{C} 4 \mathrm{C}$} & 150 & 2 \\
\hline & $\begin{array}{l}\text { Traduzir de } \\
\text { linguagem } \\
\text { natural para } \\
\text { algébrica } \\
\end{array}$ & $\begin{array}{c}\text { Traduzir de } \\
\text { linguagem } \\
\text { algébrica para } \\
\text { natural }\end{array}$ \\
\hline C4D & 88 & 27 \\
\hline
\end{tabular}

Fonte: Lucas (2010)
Espanha

\begin{tabular}{|c|c|c|}
\hline & $\begin{array}{l}\text { TAREFA } \\
\text { DIRETA }\end{array}$ & $\begin{array}{c}\text { TAREFA } \\
\text { INVERSA }\end{array}$ \\
\hline & $\begin{array}{c}\text { Representar } \\
\text { graficamente a } \\
\text { partir da expressão } \\
\text { analítica }\end{array}$ & $\begin{array}{c}\text { Escrever } \\
\text { analiticamente uma } \\
\text { função a partir do } \\
\text { gráfico }\end{array}$ \\
\hline \multirow[t]{2}{*}{ C4A } & 156 & 35 \\
\hline & $\begin{array}{l}\text { Resolver uma } \\
\text { equação } \\
\text { polinomial }\end{array}$ & $\begin{array}{c}\text { Determinar uma } \\
\text { equação polinomial } \\
\text { dadas as raízes }\end{array}$ \\
\hline \multirow[t]{2}{*}{ C4B } & 237 & 29 \\
\hline & $\begin{array}{c}\text { Resolver um } \\
\text { sistema de } \\
\text { equações lineares }\end{array}$ & $\begin{array}{c}\text { Determinar um } \\
\text { sistema de equações } \\
\text { lineares dadas as } \\
\text { soluções }\end{array}$ \\
\hline \multirow[t]{2}{*}{ C4C } & 516 & 1 \\
\hline & $\begin{array}{c}\text { Traduzir de } \\
\text { linguagem natural } \\
\text { para algébrica }\end{array}$ & $\begin{array}{c}\text { Traduzir de } \\
\text { linguagem algébrica } \\
\text { para natural }\end{array}$ \\
\hline C4D & 145 & 40 \\
\hline
\end{tabular}

Fonte: Fonseca (2004)

Indubitavelmente, os dados refletem um maior número de exercícios propostos referentes à tarefa direta do que relativos à tarefa inversa. Por conseguinte, as técnicas inversas estão ausentes nos livros didáticos. Nos dois países é observada, na conjetura C4C, uma grande discrepância entre o número de exercícios relativos à tarefa direta resolver um

\footnotetext{
${ }^{8}$ Limitar-nos-emos às funções afins e quadráticas.

${ }^{9}$ Nestas conjeturas, C4C e C4D, apenas utilizamos os livros do $3 .^{\circ}$ ciclo e ESO.
} 
sistema de equações lineares (150 em Portugal e 516 em Espanha) e à tarefa inversa escrever um sistema de equações lineares dadas as soluções (2 em Portugal e 1 em Espanha).

\section{C5. Ausência de situações abertas de modelagem}

$\mathrm{Na}$ investigação efetuada por Fonseca sobre os manuais espanhóis, em 2004, foram estabelecidas conjeturas referentes à existência de poucas situações abertas que exigem um trabalho de modelagem usando inequações $(\mathbf{C 5 A})$, derivadas $(\mathbf{C 5 B})$ ou integrais $(\mathbf{C 5 C})$. Os resultados foram os seguintes:

Tabela 5 - Resultados da conjetura 5 em Espanha

\begin{tabular}{|c|c|c|c|}
\hline & $\begin{array}{c}\text { Tipos de tarefas } \\
\text { (Problemas sobre) }\end{array}$ & Total & $\begin{array}{c}\text { Incluem alguma } \\
\text { etapa de modelagem }\end{array}$ \\
\hline C5A & inequações & 152 & 22 \\
\hline C5B & derivadas & 1957 & 176 \\
\hline C5C & integrais & 1887 & 132 \\
\hline
\end{tabular}

Fonte: Fonseca (2004)

Observou-se que, quando porventura surge alguma etapa de modelagem matemática esta reduz-se apenas à manipulação do modelo apresentado no enunciado do problema. Das cerca de 4000 tarefas analisadas, verificou-se que nenhuma exige que o aluno escolha as variáveis apropriadas para modelar um dado sistema (matemático ou extra-matemático).

Neste trabalho de investigação, a contagem das tarefas foi efetuada de forma diferente, porque os manuais portugueses apresentam muitos problemas de modelagem. Consequentemente, consideramos que seria interessante, mais do que verificar a existência/ausência de situações de modelagem, contar os problemas que requerem a construção do modelo, os que sugerem apenas a manipulação do modelo já construído e os que englobam os dois processos. Pretendemos assim, nesta conjetura, testar uma única hipótese:

Nas PM estudadas no Secundário existem poucas situações abertas que requerem um trabalho simultâneo de construção e manipulação de um modelo.

Quadro 2 - Hipótese específica para o sistema de ensino português (LUCAS, 2010)

Analisamos diversos temas nos manuais escolares portugueses (derivada, percentagens, funções polinomiais e funções definidas por ramos) e contamos os problemas que incluem a fase de construção e/ou manipulação do modelo. Na tabela seguinte registramos os resultados:

Tabela 6 - Resultados da conjetura 5 em Portugal - Fonte: Lucas (2010) 


\begin{tabular}{|c|c|c|c|c|c|c|}
\cline { 2 - 7 } \multicolumn{1}{c|}{} & \multicolumn{2}{c|}{$\begin{array}{c}\text { Construção do } \\
\text { modelo }\end{array}$} & \multicolumn{2}{c|}{$\begin{array}{c}\text { Manipulação do } \\
\text { modelo }\end{array}$} & \multicolumn{2}{c|}{$\begin{array}{c}\text { Construção e } \\
\text { Manipulação do } \\
\text { modelo }\end{array}$} \\
\hline N. $^{0}$ de Problemas & não inclui & inclui & não inclui & inclui & $\begin{array}{c}\text { não } \\
\text { inclui }\end{array}$ & inclui \\
\hline Derivadas & 96 & 51 & 20 & 127 & 96 & 51 \\
\hline Percentagens & 69 & 1 & 69 & 1 & 69 & 1 \\
\hline $\begin{array}{c}\text { Funções } \\
\text { polinomiais }\end{array}$ & 27 & 55 & 24 & 58 & 49 & 33 \\
\hline $\begin{array}{c}\text { Funções por } \\
\text { ramos }\end{array}$ & 12 & 5 & 3 & 14 & 12 & 5 \\
\hline
\end{tabular}

De todos os temas analisados observou-se, à exceção das funções polinomiais, um maior número de problemas que não incluem a construção do modelo do que os que incluem essa tarefa. É interessante salientar a grande diferença de resultados nos temas Derivadas e Percentagens. Note-se que dos 147 problemas sobre derivadas apenas 20 não incluem uma tarefa de modelagem. Esse grande número de problemas com derivadas refere-se, essencialmente, a

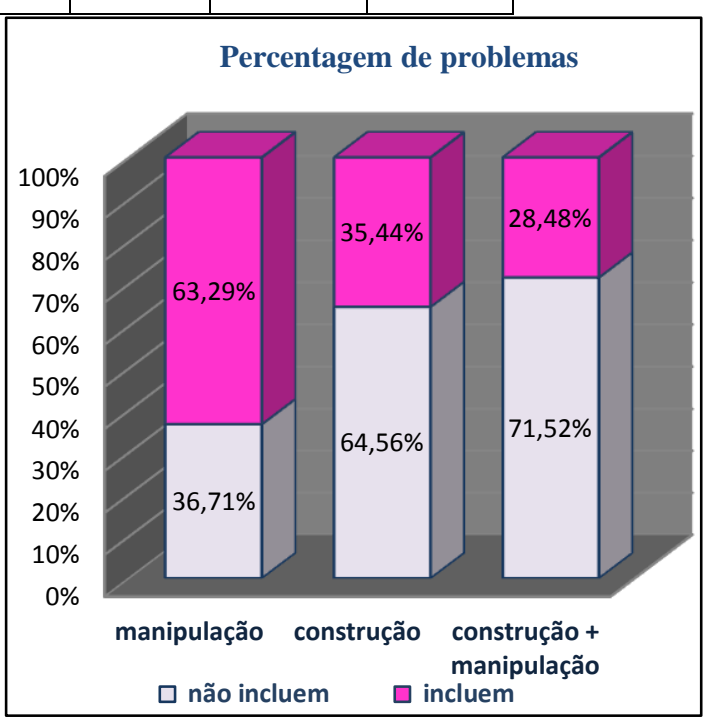
problemas de otimização. Assim, concluímos que a

Gráfico 1 - Comparação da percentagem de tarefas que incluem apenas a construção de modelos com a percentagem das que incluem apenas a sua manipulação (LUCAS, 2010).

maioria dos problemas que incluem alguma etapa de modelação, essa corresponde à manipulação do modelo. Dos 316 problemas analisados, em 204 problemas não está presente a tarefa de construir o modelo, apenas incluem a manipulação. Os dados ${ }^{10}$ representados no Gráfico 1 refletem claramente a diferença de percentagem de problemas que incluem a manipulação de um modelo já existente $(63,29 \%)$ e de problemas que ainda incluem a construção do modelo $(28,48 \%)$. Concluímos que, globalmente, nos problemas estudados está mais presente a tarefa de manipulação do modelo do que a tarefa relacionada com a sua construção.

\section{Conclusões}

Relativamente à primeira conjetura da rigidez da Matemática no ensino secundário, observamos que os dados revelam a escassez de exercícios nos livros didáticos que permitam

${ }^{10}$ Resultantes da reunião de todos os dados relativos aos quatro temas. 
que o aluno manipule uma técnica específica usando nomenclaturas incomuns. Assim, concluímos que as técnicas matemáticas tendem a identificar-se com os objetos ostensivos (símbolos, palavras e gráficos) que se utilizam para descrevê-las e implementá-las. Esta uniformidade na nomenclatura e a pouca variedade de tarefas relacionadas com uma determinada organização matemática provocará um grande obstáculo para os alunos de Matemática no primeiro ano da Universidade. Estes dados são coerentes com as respostas proporcionadas pelos estudantes ao questionário citado (LUCAS, 2010) e elaborado para detectar a incidência de diferentes aspetos da rigidez na atividade matemática escolar dos estudantes. A partir dos dados obtidos referentes ao primeiro aspeto da rigidez da Matemática, concluímos que as técnicas utilizadas no ensino secundário dependem fortemente da nomenclatura, uma vez que, bastou simplesmente substituir no questionário o símbolo representativo da incógnita por um menos habitual para que o número de respostas incorretas e em branco dos estudantes aumentasse consideravelmente.

A segunda conjetura está relacionada com o fato de que o conjunto de regras que regem a repartição das responsabilidades entre o professor e os estudantes do secundário, não atribui ao aluno a responsabilidade de interpretar o resultado obtido após a aplicação de uma técnica matemática. Os dados suportam a hipótese de que a atividade matemática no secundário é essencialmente prático-técnica e raramente atinge o nível tecnológico. Os resultados do estudo exploratório efetuado nos manuais escolares confirmam que, no secundário, não existem, praticamente, tarefas institucionalizadas que tenham como objetivo interpretar o funcionamento ou o resultado da aplicação de uma técnica. As respostas ao questionário mencionado (LUCAS, 2010), correspondentes a esta segunda conjetura mostram que os alunos manifestam dificuldades nas tarefas em que intervém o bloco tecnológicoteórico, em particular, na interpretação da atividade matemática.

A terceira conjetura da rigidez das praxeologias resume-se na existência de uma técnica privilegiada associada a cada tarefa matemática do secundário. Significa que o contrato didático não permite que o aluno tenha a responsabilidade de decidir, do conjunto das diversas técnicas úteis para resolver uma tarefa, qual é a mais econômica ou a mais fiável. Os dados empíricos extraídos dos manuais permitem explicar por que razão os estudantes nunca comparam o custo de duas técnicas diferentes para decidir, em cada caso, qual é a mais adequada, uma vez que é uma atividade praticamente ausente nos livros didáticos. E, efetivamente, nas suas respostas às tarefas do questionário (LUCAS, 2010), os alunos mostram uma forte tendência em utilizar para cada tarefa uma técnica privilegiada, mesmo no 
caso em que, o uso de uma técnica alternativa pudesse simplificar bastante a realização da tarefa.

A falta de inversão das técnicas representa a quarta conjetura da rigidez das organizações matemáticas do secundário. Os dados empíricos extraídos dos livros didáticos mostram que, em geral, a inversão das tarefas e o uso das correspondentes técnicas inversas são atividades relativamente ausentes na Matemática escolar. Explica-se assim, por que razão os estudantes não invertem uma técnica quando lhes propõem a tarefa inversa e porque basta alterar, por exemplo, a determinação das soluções de uma equação para a determinação da equação sabendo as soluções para que o número de respostas corretas decresça significativamente tal como se observa na análise das respostas ao questionário (LUCAS, 2010).

Finalmente, observamos a baixa frequência de situações de modelagem nos manuais, em particular, questões que envolvem simultaneamente a construção e a manipulação de modelos que traduzem situações reais (quinta conjetura). Um dos principais indicadores do grau de flexibilidade de uma PM local é precisamente a existência de tarefas matemáticas abertas de modelagem. A sua importância como indicador da flexibilidade provém do fato de que a existência de uma verdadeira atividade de modelagem matemática (no sentido que se descreve em BARQUERO, 2009; RUIZ-MUNZÓN, 2010 e SERRANO, 2013) pressupõe um grau de flexibilidade das técnicas e, ainda mais, pressupõe que as organizações matemáticas pontuais atingiram um determinado grau de articulação. No entanto, observando os resultados empíricos provenientes dos livros didáticos, explica-se perfeitamente as dificuldades dos estudantes quando no questionário citado (LUCAS, 2010) são confrontados com tarefas que envolvem a construção e a manipulação de modelos que traduzem situações reais.

É importante acentuar que os diferentes aspectos da rigidez das PM escolares não são independentes entre si. Em particular, a ausência de uma atividade matemática de construção e manipulação de modelos matemáticos para responder a questões, que podem surgir tanto em contextos matemáticos como extra-matemáticos, longe de ser independente dos restantes aspectos da rigidez das PM escolares, está fortemente condicionada por eles. De fato, a excessiva dependência da nomenclatura, o uso cego de técnicas sem nenhum tipo de interpretação do resultado obtido, a ausência de questionamento da economia das técnicas matemáticas e do seu domínio de validade e, em particular, a escassíssima presença de técnicas alternativas para realizar uma determinada tarefa e para realizar as tarefas inversas às escolarmente habituais, constituem restrições ecológicas que dificultam e, até mesmo, impedem a vida da modelagem matemática no ensino secundário. Com efeito, formulada uma 
questão num sistema matemático ou extra-matemático, a modelagem de tal sistema (escolha das variáveis relevantes que caracterizam o sistema, construção de um modelo matemático, trabalho dentro do mesmo e correta interpretação de tal trabalho e dos resultados obtidos, reformulação do modelo para responder a novas questões, análise do ajuste do modelo ao sistema, etc.) requer um trabalho com organizações matemáticas flexíveis (no sentido de não rígidas) e articuladas.

Em resumo, podemos concluir em primeiro lugar que a rigidez, e a correspondente atomização das PM escolares, constitui um fenômeno didático de caráter institucional visto que, com tonalidades diferentes e em diferentes graus, se evidencia quer nos manuais escolares espanhóis quer nos portugueses e, paralelamente, a atividade matemática dos alunos é completamente coerente com os dados recolhidos desses manuais. Na verdade, alunos com diferentes culturas, sociedades, tradições e também, diferentes níveis educativos manifestam um comportamento semelhante ao responder a um questionário relativo à rigidez e atomização de certas organizações matemáticas, o que induz-nos a acreditar que, no que se refere à rigidez e atomização, o tipo de atividade matemática proposta em Portugal e em Espanha é similar (apesar de algumas diferenças na abordagem) e também tem consequências comparáveis.

A segunda conclusão que queremos destacar refere-se a uma das principais consequências do fenômeno da rigidez e consequente atomização das PM escolares. Trata-se da escassa presença da atividade de modelagem matemática nas instituições escolares e, em particular, no ensino secundário. Segundo a TAD, postulamos que as dificuldades objetivas mais imediatas que atingem qualquer tentativa de implementar, de forma generalizada, a atividade de modelagem nos sistemas de ensino são provenientes do fenômeno institucional de rigidez que descrevemos. Existem muitas outras restrições que resultam de mais além da estrutura das praxeologias matemáticas escolares tais como: a forma de interpretar a atividade matemática por parte das instituições escolares (o que denominamos por modelo epistemológico dominante em tais instituições) e, correlativamente, a forma de organizar o estudo das mesmas, que denominamos por modelo didático dominante. Os trabalhos de Barquero (2009), Ruiz-Munzón (2010) e Serrano (2013) analisam detalhadamente estas restrições genéricas e a sua incidência na vida escolar da modelagem matemática.

Dada a importância de ensinar as matemáticas como ferramenta de modelagem, tal como tem sido destacado por inúmeras pesquisas em educação matemática (KAISER et al. 2006, BLUM et al. 2008), é essencial descrever claramente as condições necessárias para que este tipo de atividade matemática possa viver com normalidade numa determinada instituição, 
assim como, as restrições que atualmente a tornam difícil e até mesmo a impedem. O nosso trabalho, ao descrever as restrições mais imediatas ou específicas que sofre a modelagem matemática, situa-se precisamente neste ponto.

Com base na análise de tais restrições e como resposta a este problema de grande alcance, que poderíamos designar por problema didático da difusão escolar da modelagem matemática, pesquisas recentes no âmbito da TAD propuseram a utilização de um novo dispositivo didático, os percursos de estudo e investigação (PEI) (CHEVALLARD, 2005) para introduzir em sala de aula os processos de modelagem. Considera-se que um PEI vem gerado pelo estudo de uma questão viva com um forte poder gerador, capaz de levantar um grande número de questões derivadas. $\mathrm{O}$ estudo destas questões conduz à construção, pela comunidade do estudo, das respostas provisórias que irão demarcar o mapa dos possíveis percursos e os seus limites. Os PEI recuperam assim, a verdadeira relação entre perguntas e respostas (dando prioridade às questões) que está na origem da construção de todo o conhecimento científico.

Dentre as funções dos PEI relacionadas com a implantação das condições necessárias para que a modelagem matemática possa viver normalmente nas instituições escolares, destacamos as seguintes: (a) os PEI possibilitam que o processo de estudo tenha uma certa continuidade no tempo e rompa com a atomização das questões matemáticas, e (b) os PEI situam o questionamento tecnológico como um motor do processo de estudo ao provocar a necessidade de reestruturar, modificar, corrigir e interpretar os modelos estudados mediante a progressiva ampliação das hipóteses sobre o sistema e a correlativa construção de outros modelos mais amplos e complexos.

Por tudo isto, os PEI constituem um dispositivo didático eficaz para começar a superar o fenômeno da rigidez e a atomização da Matemática escolar, instaurando assim as condições mínimas para viabilizar a modelagem matemática.

\section{Referências}

ARTIGUE, M. De la compréhension des processus d'apprentissage a la conception de processus d'enseignement, Documenta Mathematica. Bielefeld (Germany). Extra Volume ICM, III, p. 723733. 1998. 
BARQUERO, B. Ecología de la modelización matemática en la enseñanza universitaria de las matemáticas. 2009. 537 p. Tesis (Doctoral en Matemáticas) - Departamento de Matemática, Universitat Autónoma de Barcelona, Barcelona, 2009.

BLUM, W.; GALBRAITH, P. L.; HENN, H.-W.; NISS, M. Modelling and applications in mathematics education: the 14th ICMI study. New ICMI Study Series Volume 10. ZDM The International Journal on Mathematics Education, Karlsruhe (Germany). v. 40, n. 2, p. 337-340, May. 2008.

BOSCH, M.; FONSECA, C.; GASCÓN, J. Incompletitud de las organizaciones matemáticas locales en las instituciones escolares. Recherches en Didactique des Mathématiques, Grenoble: Sauvage, v. 24, n. 2-3, p. 205-250, $4^{\circ}$ trimestre. 2004.

BROUSSEAU, G. Theory of Didactical Situations in Mathematics. Didactique des mathématiques, 1970-1990. (N. Balacheff, R. Sutherland, \& V. Warfield, Eds.) Dordrecht: Kluwer Academic Publishers, 1997.

CHEVALLARD, Y. L'analyse des pratiques enseignantes en théorie anthropologique du didactique. Recherches en Didactique des Mathématiques, Grenoble: La Pensée Sauvage, v. 19, n. 2, p. 221 266, Août. 1999.

CHEVALLARD, Y. La place des mathématiques vivantes dans l'éducation secondaire : transposition didactique et nouvelle épistémologie scolaire. In : DUCOURTIOUX, C.; HENNEQUIN, P.-L. (Ed.). La place des mathématiques vivantes dans l'enseignement secondaire. Publications de l'APMEP,. Paris: APMEP. 2005. p. 239-263

CHEVALLARD, Y.; BOSCH, M.; GASCÓN, J. Estudiar matemáticas. El eslabón perdido entre la enseñanza y el aprendizaje, Barcelona: ICE/Horsori, 1997. (Existe tradução em português: Estudar Matemáticas. $O$ elo perdido entre o ensino e a aprendizagem, Porto Alegre (Brasil) Artmed Editora, 2001).

DREYFUS, T. Advanced Mathematical Thinking Processes, In David Tall (Ed.). Advanced Mathematical Thinking, Dordrecht: Kluwer, 1991. p. 25-41.

DUBINSKY, E. Reflective Abstraction in Advanced Mathematical Thinking, In: David Tall (Ed.). Advanced Mathematical Thinking, Dordrecht: Kluwer, 1991. p. 95-123.

FONSECA, C. Discontinuidades matemáticas y didácticas entre la Secundaria y la Universidad. 2004. 263 p. Tesis (Doctoral en Ciencias Matemáticas) - Departamento de Matemática Aplicada I, Universidad de Vigo, Vigo, 2004.

GASCÓN, J. Evolución de la didáctica de las matemáticas como disciplina científica. Recherches en Didactique des Mathématiques, Grenoble: La Pensée Sauvage, v. 18, n. 1, p. 7-34, Avril. 1998.

GASCÓN, J. From the cognitive to the epistemological program in the didactics of mathematics: two incommensurable scientific research programs. For the learning of mathematics, v. 23, n. 2, p. 44$55,2003$.

GRAY, E.; TALL, D. Duality, Ambiguity and Flexibility: A Proceptual View of Simple Arithmetic, Journal for Research in Mathematics Education, Reston (USA). v. 26, nº 2, p. 115-141. 1994.

KAISER, G.; BLOMHØJ, M.; SRIRAMAN, B. A brief survey of the state of mathematical modeling around the world. ZDM The International Journal on Mathematics Education, Karlsrhue:

Springer. v. 38, n.3, p. 212-213, June. 2006.

LUCAS, C. Organizaciones Matemáticas Locales Relativamente Completas. 2010. 256 p. Tesina (Diploma de Estudios Avanzados: Programa Doctoral de Técnicas Matemáticas Avanzadas y sus Aplicaciones) - Departamento de Matemática Aplicada I, Universidad de Vigo, Vigo, 2010. 
PIAGET, J.The Principles of Genetic Epistemology, London: Routledge and Kegan Paul, 1972.

PONTE, J. P.; MATOS, J. F. Processos cognitivos e interacções sociais nas investigações matemáticas. In: P. Abrantes, L. C. Leal,; J. P. Ponte (Ed.). Investigar para aprender matemática, Lisboa: APM e Projecto MPT, 1996. p. 119-137.

RUIZ-MUNZÓN, N. La introducción del álgebra elemental y su desarrollo hacia la modelización funcional. 2010. 2 v. Tesis (Doctoral en Matemáticas) - Departamento de Matemática, Universitat Autónoma de Barcelona, Barcelona, 2010.

SERRANO, L. La modelización matemática en los estudios universitarios de economía y empresa: análisis ecológico y propuesta didáctica. 2013. 363 p. Tesis (Doctoral en Estadística Aplicada) - Departamento de Estadística Aplicada, Universitat Ramon Llull, Barcelona, 2013.

SFARD, A. On the Dual Nature of Mathematical Conceptions: Reflections on processes and objects as different sides of the same coin, Educational Studies in Mathematics, v. 22, n ${ }^{\circ}$ 1, p. 1-36, February. 1991.

SILVA, A.;VELOSO, E.; PORFÍRIO, J.; ABRANTES, P. O Currículo de Matemática e as Actividades de Investigação. In: P. ABRANTES. J. P. PONTE, H. FONSECA; L. BRUNHEIRA (Org.). Investigações matemáticas na aula e no currículo. Lisboa: APM e Projeto MPT, 1999. p. 69-85.

TALL, D. Functions and Calculus, In: A. J. BISHOP et al. (Ed.). International Handbook of Mathematics Education, Dordrecht: Kluwer, 1996. p. 289-325.

Submetido em Junho de 2013. Aprovado em Agosto de 2013. 